\title{
Review of Application Research of Expert System and Neural Network in Credit Risk Evaluation
}

\author{
Mu ZHANG \\ School of Finance, Guizhou University of Finance and \\ Economics, Guiyang Guizhou 550025, China \\ rim_007@163.com
}

\author{
Li DONG \\ School of Finance, Guizhou University of Finance and \\ Economics, Guiyang Guizhou 550025, China \\ Guizhou Institute for Urban Economics and \\ Development, Guizhou University of Finance and \\ Economics, Guiyang Guizhou 550025, China \\ Guizhou Institution for Technology Innovation \& Entre \\ preneurship Investment, Guizhou University of Finance \\ and Economics, Guiyang Guizhou 550025, China \\ 954388937@qq.com
}

\begin{abstract}
Theoretical and empirical research on. credit risk evaluation plays an important role for enterprises in reducing transaction costs, enhancing competition ability and creating a good competition environment. At present, in view of the application of expert system and neural network in artificial intelligence method in enterprise credit risk evaluation, research abroad mainly focuses on the innovative application of expert system and neural network; and the domestic research mainly focuses on the advantages and disadvantages, the accuracy and the feasibility of expert system and neural network. In this paper, the research status of expert system and neural network at home and abroad is discussed in detail so that the development of artificial intelligence theory and practice is promoted.
\end{abstract}

Keywords-credit risk; credit risk evaluation; expert system; neural network; artificial intelligence

\section{INTRODUCTION}

Since 1980s, with the development of financial liberalization and globalization and the increased volatility in international financial markets, international commercial banks and investors are facing unprecedented challenges of credit risk. All kinds of new credit risk evaluation methods emerge in endlessly, and credit risk management theory and technology are improving day by day[1]. Generally speaking, the methods of credit risk assessment have undergone a gradual process from simple to complex, from qualitative analysis to quantitative calculation and from credit risk evaluation of single asset to credit risk evaluation of portfolio[2]. The traditional credit risk evaluation method, multivariate statistical analysis method, artificial intelligence methods and models of credit risk measurement appeared successively.

Artificial Intelligence (AI) is a new technological science that studies, extends and develops theories, methods, techniques and application systems. Artificial Intelligence is a branch of computer science that tries to understand the nature of intelligence and produces a new intelligent machine that responds in a similar way to human intelligence. Since the birth of artificial intelligence, the theory and technology have become more and more mature, and the field of application has also been expanding. Now, it has been gradually applied to credit risk evaluation. In this paper, the application status at home and abroad of expert system and neural network in credit risk evaluation is discussed in detail so that the application of artificial intelligence methods in credit risk assessment are promoted.

\section{EXPERT SYSTEM}

The expert system is an intelligent computer program that uses knowledge and reasoning to reproduce the reasoning process of an expert solving problems. It becomes an expert decision-making tool, or provides professional advice for non professional decision makers.Explanation function, flexibility and learning function are the three main functions of expert system. Foreign research scholars Messier and Hansen(1988)[3]broken the limitations of the direct method, and put forward a heuristic way of acquiring knowledge. And they from the angle of knowledge acquisition studied the application of expert system in the field of credit risk analysis. First, they extracted the features according to expert examples. Then, they used the heuristic algorithms to obtain production rules, and selected concept learning algorithms to extract generic results from known categories. On this basis, a production system is established, and the rules in the system are used to evaluate the credit of the new sample. The conclusions of the study shows that the expert system is better than discriminant analysis and group decision making. Bing Guo,Shidong Liang, and Zhaoben Fang(2001)[4] were back in the history of the development of consumer credit evaluation, analyzed the main problems in the process of establishing China's consumer credit evaluation system, and also introduced the present application of the consumer credit evaluation method including artificial intelligent methods such as expert system,neural network and etc. At home, Jian Jin and 
Chengde Lin(2003)[5]combined artificial neural network and expert system to evaluate the credit of commercial banks. Taking a hybrid expert system ECAMES as an example, the design and implementation of a hybrid expert system model is described. Baoan Yang and Ming Zhu(1999, 2006)[6-7] combined artificial neural network (ANN) and expert system (ES) to study loan risk management decision for realizing the risk prevention and control and improving the credit management level of commercial banks and promoting the management decisions of banks scientific and intelligent. Based on the analysis of the advantages and disadvantages of traditional expert methods, Peng Zhang, Yongquan Ceng, Chaoyuan Yue(2013)[8] proposed a new expert method for credit risk measurement. The new method uses the principle of cluster analysis to eliminate interference from individual expert opinions so as to conform to the majority principle. The new expert method enables the experts to have the additivity and the comparability for the borrower credit score value. So it overcomes the shortcomings of traditional expert methods, such as subjective randomness and inconsistency. Jin Xu and Zhenfa Qi(2013)[9] put forward a supplier credit rating analysis index system and evaluate it by artificial intelligence neural network. Finally, based on the reset algorithm, a dynamic neural network expert system is constructed.

\section{NEURAL NETWORK}

Neural network(NN) is a computer system with pattern recognition ability. It has the characteristics of selforganization, self adaptation and self-learning. The knowledge of $\mathrm{NN}$ is encoded in the whole weight network, and it is in distributing storage and has a certain fault tolerance. NN does not require strict distribution of sample data, and nor does it require a detailed description of the functional relationship between independent variables and dependent variables[10]. The above characteristics of NN have greatly promoted the application of $\mathrm{NN}$ in credit risk evaluation.Model neural network, probabilistic neural network, extended learning vector quantizer and multilayer perceptron have been applied effectively. In the credit risk assessment, the classification function of NN is mainly used. The basic train of thought is that: firstly, a set of factors that influence the classification of samples are given as input to $\mathrm{NN}$. and then, the NN credit risk assessment model is formed through the training of a supervisor or no supervisor, and the credit category of the new sample is distinguished.

Desai et al(1996)[11]introduced the model neural network (MNN) into credit risk analysis, and compared it with the multilayer perceptron (MLP) and the multiple discriminant analysis (MDA). Tyree and Long(1995)[12]studied the application of probabilistic neural networks (PNN) in credit risk assessment.The results showed that the discriminant accuracy of PNN was significantly higher than that of MDA. The extended learning vector quantizer (ELVQ) was applied to credit risk assessment by Poddig(1995)[13]. ELVQ has the advantages of quick training,easy to grasp, relatively simple structure and so on. It is convenient for commercial banks to adjust in time so as to meet the immediate change of borrowers' groups.
Because of the theory research of multi layer perceptron (MLP) is relatively mature, and the deletion technology in the network construction is more perfect than other neural networks. Therefore, in many neural network methods, MLP is the most widely used method of credit risk assessment. At present, in the analysis of credit risk, there are many discussions about the neural network based BP algorithm. There are mainly foreign literatures such as Salchenberger et al(1992)[14], Coats and Fant(1993)[15], Altman and Marco(1994)[16], Kerling(1995)[17], Yurt(1995)[18] and etc. And there also are mainly domestic literatures such as Chunfeng Wang(1999)[19], Xionghua Chen(2002)[20], Xingde Zhu and Tiejun Feng(2003)[21], Desheng Wu and Liang Liang(2003, 2004)[22-24, Peng Zou(2005)[25], Guoqing Liu and Honglei Wang(2009)[26], Xiaofeng Li and Jiuping $\mathrm{Xu}(2010)[27]$ and etc.

At home, Shuang Pan and Yuan Huang(2010)[28] used the improved wavelet neural network model to establish the credit risk evaluation system of commercial banks, and realized the nonlinear mapping of bank information, financial indicators and risk rating.In order to improve the accuracy of commercial bank credit risk assessment, Jinhua Zhu(2011)[29] proposed the commercial bank credit risk assessment portfolio model which is a combination model of rough set theory (RS) and BP neural network (BPNN). In order to combine the advantages of different single models and solve the problem of accuracy and stability of credit risk early-warning model, Xinhong Zhang, Ruixiao Wang(2011)[30] selected the traditional mathematical statistical model including Logistic regression model, artificial intelligence model RBF and neural network model to establish a combination forecasting model. On the basis of expert evaluation index, in order to improve the accuracy of enterprise credit risk assessment, Juncai Shen and Jihong $\mathrm{Xu}(2012)$ [31] put forward a method of enterprise credit risk assessment based on BP neural network.Using the field investigation data of three rural credit cooperatives in Yangling District in 2009, Yao Shuqiong and Qiang Junhong(2012)[32] conducted an empirical study on the credit risk assessment of Rural Micro Credit and MATLAB7.0 software is used by them to establish the farmer credit risk evaluation model of 8 - 14 - 1 structure BP neural network. According to the enterprise credit evaluation index system established by the Guiding standards and norms of enterprise credit evaluation in Zhejiang Province, Yusheng Lou(2013)[33] established the enterprise credit risk evaluation model of fuzzy neural network. Wen $\mathrm{Xu}$, Tianxing Zhu, Mingsheng Xu(2013)[34] introduced the characteristics of SME credit risk and the steps of model development. And based on Logistic regression model and neural network model, the listed SMEs credit risk rating model is constructed by they.Finally, they introduced the verification and calibration methods of the above model.Xunyan Jiang(2014)[35]put forward the early warning system of BP neural network to warn the credit risk. Xiangdong Liu and Weiqing Wang(2015)[36] took the financial data of China's A shares of 325 listed companies in 2011 and 2012 as samples, and used Bayesian discriminant analysis, Logistic regression model and BP neural network 
model to identify the credit risk, and then compared the accuracy, prediction ability and stability of the three models. According to the characteristics of the P2P network lending platform, and taking the individual credit rating of the platform borrower as the prediction output target, Liping Song,Likun Zhang,Wei $\mathrm{Xu}$ (2015)[37] determined the personal credit risk evaluation index and created the BP neural network model for better understanding the borrower's credit status.Starting with the analysis of the credit risk of the borrower of the network lending platform, Jianglin Huo, Surong Liu(2016)[38] established the credit risk evaluation index system of the borrower and built a credit risk assessment model based on artificial neural network. Based on the actual credit data of small and micro enterprises in a commercial bank from 2008 to 2013, Xiao Binqing, Yang Yang, Yu Zhe, Shen Caisheng (2016) [39] used linear discriminant analysis, two logistic regression and ten BP neural network models based on different learning algorithms to build internal credit rating model, and macroeconomic variables are added to the rating index system to further enhance the robustness of measurement risk.

\section{BRIEF COMMENTS}

Expert system is an important branch of artificial intelligence in early stage, it can be seen as a kind of computer intelligent system of having special knowledge and experience, and knowledge representation and knowledge reasoning techniques in artificial intelligence are usually used to simulate complex problems that can usually be solved by domain experts. Compared with other methods in the application of credit risk assessment, expert system method has three major functions such as explanation function, flexibility and learning. Knowledge acquirement is a bottleneck of developing expert system, and the reasoning method determines the intelligence level of expert system.The research progress of knowledge acquisition and reasoning method determines the application prospect of expert system in the field of credit risk analysis.

The neural network is a mathematical model of imitating the behavior of the animal neural network and distributing the parallel information processing. The neural network has the characteristics of self-organization, self adaptation and self-learning. The distribution of sample data is not strictly required, and there is no need to specify the functional relationship between independent variables and dependent variables. The advantages of neural network is that the distribution of sample data is not strict and it has the ability to deal with nonlinear problems, And the output is somewhere between 0 and 1 . In the case of credit risk, that figure is behalf of the probability of default. There are three drawbacks of the neural network. First, there is the problem of "black box", it is unable to know its way of operation and lacks of explanation and lacks of solid statistical theories and foundations. Second, the algorithm is easy to form local minima without global optimization. Finally, the algorithm has many iterations and the convergence speed is very slow. Therefore, NN technology in practice is often used as an auxiliary method of "verification".

\section{ACKNOWLEDGMENT}

This work was financially supported by National Natural Science Foundation of China (71263011).

\section{REFERENCES}

[1] Ling Zhang,Jialin Zhang.Development trend of credit risk assessment methods [J].Projections,2000,(4):72-75.

[2] Konglin Ke,Chunxi Zhou.Review of credit risk assessment methods for commercial banks [J].Business Economics and Management ,2005,(6):55-60.

[3] Messier W F, Hansen J V. Inducing rules for expert system development an example using default and bankruptcy data[J]. Management Science,1988,34(12):1403-1415.

[4] Bing Guo,Shidong Liangand Zhaoben Fang.Review of consumer credit evaluation analysis[J].System Engineering,2011,(6):9-15.

[5] Jian Jin,Chengde Lin.Design and implementation of credit rating system model based on hybrid expert system [J].Computer Application ,2003,23(4):81-83.

[6] Baoan Yang,Ming Zhu.Bank loan risk management based on neural network and expert system [J].System Engineering,Theory, Method, Application ,1999,8(1):7-10.

[7] Baoan Yang,Ming Zhu. Decision making research of bank loan risk management based on neural network and expert system --National Natural Science Foundation of China 79770086 Project Review [J].Journal of Management ,2006,3(4):387-390.

[8] Peng Zhang,Yongquan Ceng,Chaoyuan Yue.New expert method for credit risk measurement $[\mathrm{J}]$.Scientific and Technological Progress and Countermeasures, 2006,7:52-54.

[9] Jin Xu ,Zhenfa Qi.Supplier credit rating analysis based on dynamic neural network expert system [J]. Soft Science,2013,(10):5-8.

[10] Wei Zhang,Yushuang Li.A review of credit risk analysis of commercial banks [J].Journal of Management Science ,1998,1(3):2027.

[11] Desai V S, et al. A comparison of neural networks and linear scoring models in the credit union environment[J]. European Journal of Operational Reasearch,1996,95:23-37.

[12] Tyree E W, Long J A. Assessing financial distress with probalilistic neural networks[C]. Proceedings of the Third International Conference on Neural Networks in the Capital Market,1995.

[13] Poddig T. Bankruptcy prediction: a comparison with discriminant analysis. Neural Networks in the Capital Market[M]. John Wiley and Sons Ltd, 1995.

[14] Salchenberger Linda, et al. Neural Networks: A new tool for predicting thrift failures[J]. Decision Sciences, 1992,23:899-916.

[15] Coats P, Fant L. Recognizing financial distress patterns using a neural network tool[J]. Financial Mamagement,1993,12(3)142-155.

[16] Altman E I, Marco G. Corporate distress diagnosis: comparisons using linear discriminant analysis and neural networks (the Italian experience) [J]. Journal of Banking and Finance, 1994,18: 505-529.

[17] Kerling M. Corporate distress diagnosis: an international comparison[C]. Proceedings of the Third International Conference on Neural Networks in the Capital Market,1995.

[18] Yurt A. Neural networks in corporate failure prediction: the UK experience $[\mathrm{C}]$. Proceedings of the Third International Conference on Neural Network s in the Capital Market,1995.

[19] Chunfeng Wang,Haihui Wan,Wei Zhang.Credit risk evaluation of commercial banks based on neural network technology[J].System Engineering Theory and Practice,1999,19(9):24-33.

[20] Xionghua Chen,Chengde Lin,Wu,Ye. Evaluation of enterprise credit rating based on Neural Network,[J].Journal of System Engineering,2002,17(6):570-575. 
[21] Xingde Zhu,Tiejun Feng.Personal credit evaluation based on GA neural network [J].System Engineering Theory and Practice, 2003,23 (12): 70-75115.

[22] Desheng Wu, Liang Liang.The application of probabilistic neural network in financial early-warning demonstration [J]. China Management Science, 2003,11 (10): 173-177.

[23] Desheng Wu, Liang Liang.Genetic algorithm, neural networks of optimization and credit evaluation [J]. Chinese Management Science, 2004,12 (1): 68-74.

[24] Desheng Wu, Liang Liang.Research of credit evaluation based on VfoldCross-validation and Elman neural networks [J]. System Engineering Theory and Practice, 2004, (4): 93-98.

[25] Peng Zou,Qiang ye,Li Yijun. Self optimal neural network credit evaluation facing new basel capital accord[J]. Journal of Management, 2005,2 (4): 406-409.

[26] Guoqing Liu,Honglei Wang.GA-BP neural network model applied to credit evaluation of listed companies [J].. Economic Problem, 2009, (12): 77-80.

[27] Xiaofeng Li,Jiuping Xu.The establishment of BP neural network model for comprehensive evaluation of commercial bank customer credit [J]. Soft Science, 2010,24 (2): 110-113.

[28] Shuang Pan,Yuan Huang.Credit risk evaluation of commercial bank based on Improved Wavelet Neural Network [J].Journal of Wuhan University of Technology ,2010,(11):110-114.

[29] Zhu Jinhua.Research on portfolio model in commercial bank credit risk assessment [J].Computer Simulation, 2011, (9): 361-364.
[30] Xinhong Zhang,Ruixiao Wang.Study on credit risk early warning of Listed Companies in China [J].Macro Economic Research,2011, (1):50-54.

[31] Juncai Shen,Jihong Xu.Application Research of neural network for enterprise credit risk assessment [J].Computer Simulation,2012, (3):254-257.

[32] Yao Shuqiong, Qiang Junhong.Study on credit risk assessment of rural micro credit based on BP neural network[J].Journal of Northwest Agriculture and Forestry University, 2012,(2):78-83.

[33] Yusheng Lou.Research on enterprise credit risk assessment model based on fuzzy neural network [J]. Journal of Central South University, 2013, (5): 21-25.

[34] Wen Xu,Tianxing Zhu,Mingsheng Xu.Research on credit risk rating theory and related models of commercial banks [J]. Financial Forum, 2013, (7): 82-83.

[35] Xunyan Jiang.commercial bank credit risk early warning research based on BP neural network [J]. Financial Research, 2014, (S1): 4648.

[36] Xiangdong Liu,Weiqing Wang.Multi model comparison of credit risk identification of commercial banks in China [J].Economic Survey,2015,(6):132-137.

[37] Liping Song, Likun Zhang,Wei Xu,.personal credit risk assessment of P2P online lending,[J]. Accounting Monthly, 2015, (35): 94-96.

[38] Jianglin Huo,Surong Liu,.P2P net loan platform borrower credit risk assessment [J].Financial Development Research, 2016, (12): 43-47.

[39] Xiao Binqing,Yang Yang,Yu Zhe,Shen Caisheng.Small and micro businesses credit rating model and comparison study[J].System Engineering Journal,2016,(6): 798-807. 